\title{
High dimensional Selection with Interactions for Binary Outcome (HDSI-BO) Algorithm in Classifying Height Indicators Through Social-life and Well-being Factors
}

\author{
Ziqian Zhuang ${ }^{1}$, Wei Xu1 ${ }^{2}$ and Rahi Jain ${ }^{2}$ \\ ${ }^{1}$ Biostatistics Division, Dalla Lana School of Public Health, University of Toronto, Toronto, Canada \\ ${ }^{2}$ Biostatistics Department, Princess Margaret Cancer Research Centre, Toronto, Canada \\ E-mail: ziqian.zhuang@mail.utoronto.ca
}

Received May 15, 2020

Accepted for publication June 1, 2021

Published online September 13, 2021

\begin{abstract}
Introduction: High dimensional Selection with Interactions for Binary Outcome (HDSI-BO) algorithm can incorporate interaction terms and combine with existing techniques for feature selection. Simulation studies have validated the ability of HDSI-BO to select true features and consequently, improve prediction accuracy compared to standard algorithms. Our goal is to assess the applicability of HDSI-BO in combining different techniques and measure its predictive performance in a real data study of predicting height indicators by social-life and well-being factors.

Methods: HDSI-BO was combined with logistic regression, ridge regression, LASSO, adaptive LASSO, and elastic net. Two-way interaction terms were considered. Hyperparameters used in HDSI-BO were optimized through genetic algorithms with five-fold cross-validation. To measure the performance of feature selection, we fitted final models by logistic regression based on the sets of selected features and used the model's AUC as a measure. 30 trials were repeated to generate a range of the number of selected features and a $95 \%$ confidence interval for AUC.

Results: When combined with all of the above methods, HDSI-BO methods achieved higher final AUC values both in terms of mean and confidence interval. In addition, HDSI-BO methods effectively narrowed down the sets of selected features and interaction terms compared with standard methods.

Conclusion: The HDSI-BO algorithm combines well with multiple standard methods and has comparable or better predictive performance compared with the standard methods. The computational and time complexity of HDSI-BO is higher but still acceptable. Considering AUC as the single metric cannot comprehensively measure the feature selection performance. More effective metrics of performance should be explored for future work.
\end{abstract}

